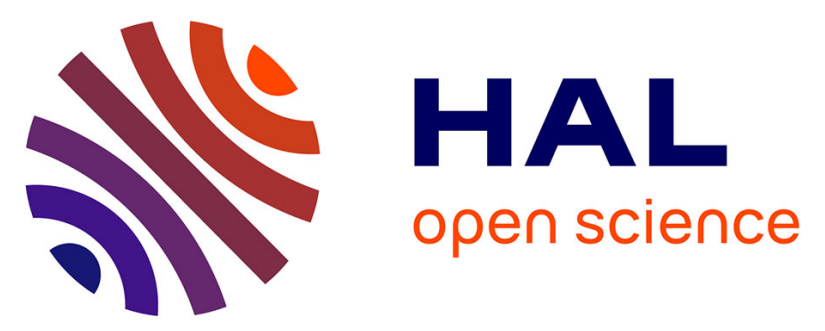

\title{
Modulation of antimicrobial resistance in clinical isolates of Enterobacter aerogenes: A strategy combining antibiotics and chemosensitisers
}

\author{
Matthew Mccusker, Daniela Alves Ferreira, Donal Cooney, Bruno Martins \\ Alves, Séamus Fanning, Jean-Marie Pagès, Marta Martins, Anne Davin-Regli
}

\section{- To cite this version:}

Matthew Mccusker, Daniela Alves Ferreira, Donal Cooney, Bruno Martins Alves, Séamus Fanning, et al. Modulation of antimicrobial resistance in clinical isolates of Enterobacter aerogenes: A strategy combining antibiotics and chemosensitisers. Journal of Global Antimicrobial Resistance, 2019, 16, pp.187-198. 10.1016/j.jgar.2018.10.009 . hal-02649582

\section{HAL Id: hal-02649582 \\ https://hal-amu.archives-ouvertes.fr/hal-02649582}

Submitted on 21 Oct 2021

HAL is a multi-disciplinary open access archive for the deposit and dissemination of scientific research documents, whether they are published or not. The documents may come from teaching and research institutions in France or abroad, or from public or private research centers.
L'archive ouverte pluridisciplinaire HAL, est destinée au dépôt et à la diffusion de documents scientifiques de niveau recherche, publiés ou non, émanant des établissements d'enseignement et de recherche français ou étrangers, des laboratoires publics ou privés.

\section{(ㄷ)(1) $\$$}

Distributed under a Creative Commons Attribution - NonCommerciall 4.0 International 
1 Modulation of antibiotic resistance in clinical isolates of Enterobacter aerogenes - a strategy combining antibiotics and chemosensitisers

3

4

7

$8{ }^{1}$ UCD Centre for Food Safety, School of Public Health, Physiotherapy and Sports Science,

9 University College Dublin, Belfield, Dublin 4, Ireland.
2UMR_MD1, U-1261, Aix Marseille Univ, INSERM, SSA, IRBA, MCT, Marseille, France.

${ }^{3}$ Department of Microbiology, Moyne Institute of Preventive Medicine, School of Genetics and Microbiology, Trinity College Dublin, the University of Dublin, Dublin 2, Ireland.

•ICON Central Laboratories; South County Business Park, Leopardstown, Dublin 18, Ireland;

"Hikma Pharmaceuticals PLC, Estrada do Rio da Mó 8 8A, 8B, 2705-906 Terrugem,

Portugal; "Department of Microbiology, Moyne Institute of Preventive Medicine, School of Genetics and Microbiology, Trinity College Dublin, Dublin 2, Ireland.

\section{*Corresponding authors}

Anne Davin-Regli. UMR_MD1, INSERM-1261, Faculté de Pharmacie. 27 Bd Jean Moulin, 13385 Marseille cedex05, France anne-veronique.regli@univ-amu.fr 
24 Marta Martins, Department of Microbiology, Moyne Institute of Preventive Medicine, School 25 of Genetics and Microbiology, Trinity College Dublin, The University of Dublin, Dublin 2,

26 Ireland. Tel: +353 1896 1194, e-mail: mmartins@ tcd.ie

27

28 Running title: Modulating antibiotic resistance in E. aerogenes clinical isolates

29

30 
31 Abstract

32 Purpose: The main focus of this study was to evaluate the antimicrobial susceptibility

33 profiles of a selected number of human clinical isolates of Enterobacter aerogenes and

34 explore the effects of selected chemosensitisers on the reversal of the resistant phenotype of

35 these isolates.

36 Methodology: This study design was accomplished by: (i) characterising several multidrug

37 resistant (MDR) E. aerogenes clinical isolates; (ii) evaluating the contribution of target gene

38 mutations to the resistance phenotype focusing on fluoroquinolones and chloramphenicol

39 only; (iii) evaluating the contribution of membrane permeability and efflux to the MDR

40 phenotype; (iv) assessing the combined action of selected antimicrobials and

41 chemosensitisers (to identify combinations with synergistic effects and able to reduce the

42 MIC); (v) understand how these combinations can modulate the permeability or efflux on

43 these isolates.

44 Results: Resistance to ciprofloxacin couldn't be totally reversed due to pre-existing mutations 45 in target genes. Chloramphenicol susceptibility was efficiently restored by the addition of the 46 selected chemosensitisers. From the modulation kinetics it was clear that phenothiazines were 47 able to increase the accumulation of the Hoescht dye.

48 Conclusions: Modulations of permeability and efflux in the presence of chemosensitisers

49 help us to propose more appropriate chemotherapeutic combinations that can set the model to 50 be used in the treatment of these and other MDR-infections.

51

52 Keywords: Chemosensitisers; efflux pump; fluoroquinolones; multidrug resistance;

53 permeability; combination therapy.

54

55 
57 Acute hospital-acquired urinary tract infections are mainly associated with Escherichia coli

58 [1]. However, pathogens such as Enterobacter, Klebsiella, some Proteus species and

enterococci can also be involved in cases of cystitis and pyelonephritis [2]. Enterobacter represents with Klebsiella the prominent enterobacterial members of the ESKAPE group that encompasses the main MDRs described in the most worrying/complicated infections in hospitals [3].

In 1999, Enterobacter aerogenes represented $48.7 \%$ of the extended-spectrum betalactamases strains, due to the dissemination of TEM-24 [4]. In 2007, the numbers decreased, being replaced by E. coli CTX-M producers $[5,6]$. It is known that during therapy, strains acquire resistance to $\beta$-lactams [4-7]. At present, E. aerogenes is the fourth most frequently isolated bacteria in the clinical setting. This prevalence has increased greatly due to the introduction of extended-spectrum cephalosporins and carbapenems [7-9]. This has contributed to the emergence of pandrug E. aerogenes isolates resistant to last-line antibiotics. Moreover, clinical strains exhibiting efflux activity are resistant to $\beta$-lactams, quinolones, tetracyclines and chloramphenicol [10-13]. Approximately 40\% of MDR clinical strains have an active efflux [14].

Clinical isolates of E. aerogenes that overexpress efflux pumps in combination with loss of porins have been reported in numerous studies $[10,15]$. The study conducted by Doumith $e t$ al. also showed that carbapenem resistance in Klebsiella and Enterobacter was exclusively due to combinations of B-lactamases with impermeability caused by loss of outer membrane proteins, where efflux was not implicated [16]. Taken these studies into account, reduction of membrane permeability through the loss of porins together with increased expression of 
81 efflux pumps can constitute a survival response involved in bacterial adaptation under stress

82 [17-18]. This is frequently linked with alteration of antibiotics or modification of the drug

83 targets in multidrug resistant (MDR) isolates [19]. In E. coli, E. aerogenes, K. pneumoniae

84 and Salmonella enterica several genes and external factors are involved in the emergence of

85 multidrug resistance [18-20]. The combined action of these mechanisms during an infection

86 confers a decrease in bacterial susceptibility ensuring dissemination and colonisation of the

87 patient and favoring the acquisition of additional mechanisms of resistance [15-18].

88

89 New antibiotics and novel approaches to treat MDR bacteria have been pursued [21]. Among

90 these, the development of chemosensitisers to return the bacteria to its susceptibility status is

91 to consider [22]. These compounds are usually of synthetic or natural origin, however a new

92 "trend" to modify existing compounds has gained strength in the last years [22-25].

93

94 The present study is focused on the modulation of efflux-mediated resistance to

95 fluoroquinolones or chloramphenicol, in clinical isolates of E. aerogenes. The strategy used

96 combined antibiotics and chemosensitisers to assess possible reversal of resistance to the

97 selected antibiotics. Efflux activity was assessed by a real-time fluorometric assay in the

98 presence of these compounds to evaluate their action on the efflux mechanism of resistance.

100

101 


\section{Materials and Methods}

\section{Media, antibiotics, chemosensitisers and dyes}

104 Mueller-Hinton (MH) broth, MH agar (MHA), Phosphate Buffered Saline (PBS) solution, 105 ciprofloxacin (CIP), chloramphenicol (CHL), cefotaxime (CTX), thioridazine (TZ),

106 chlorpromazine (CPZ), ouabain (OUB), verapamil (VER), phenylalanine-Arginine- $\beta$ -

107 naphthylamide (PAßN), Omeprazole (OME), esomeprazole (ESO), 1-(1-naphthylmethyl)-

108 piperazine (NMP), carbonyl cyanide m-chlorophenyl hydrazone (CCCP) and Hoescht dye

109 solution (stock $20 \mathrm{mM}$ ). They were all purchased from Sigma (Ireland).

\section{Bacterial strains}

112 A random analysis using nineteen E. aerogenes isolates was performed in this study. These 113 bacterial isolates were obtained from several hospital origins from the south of France in 114 order to avoid bias. Nineteen E. aerogenes clinical isolates were identified as follows:

115 EACRO, EA9AB32, EA17AB48, EA29AB151, EA26UR132, EA22SP84, EA132263, 116 EA149399, EA171450, EAPOL13200, EAART15737, EA178LAV, EABAL959, EA23214, 117 EA108418, EA118259, EA122554, EA131538, EA137454 (Table 1). The antibiotic 118 susceptible E. aerogenes ATCC13048 was used as reference strain.

120 Antibiotic Susceptibility Testing

121 Antibiotic susceptibility testing was initially determined using the VITEK-2 platform and 122 sensititre plates ${ }^{\mathrm{TM}}$ GNX-2F (TREK Diagnostic Systems, LTD; East Grinstead, West Sussex, $123 \mathrm{UK}$ ) as per the manufacturers recommendation. Disc susceptibility testing was also performed 124 and resistance reported according to the European Committee on Antimicrobial Susceptibility 125 Testing (http://www.eucast.org/antimicrobial_susceptibility_testing/breakpoints/) validated 126 for E. coli were followed, only for comparison terms [26]. For POL, the breakpoints used 
127 were the ones established by the Societe Francaise de Microbiologie, 2007. For COL the

128 results were analysed in comparison with the reference strain and other available literature

129 [27-28]. Isolates resistant to three or more different classes of antibiotics were considered

130 MDR. Isolates demonstrating mono or multiple resistances to CIP and CHL were selected for

131 further studies.

132

133 Determination of Minimum Inhibitory Concentrations (MIC)

134 The MIC of antibiotics and chemosensitisers were determined by broth microdilution method

135 [26]. All experiments were carried out in triplicate and in three separate occasions.

136

137 Assessment of respiration in the presence of chemosensitisers

138 To evaluate the effect of the chemosensitisers on the respiration of the isolates, assays were

139 prepared in 96-well plates as for MIC determination with media containing a tetrazolium

140 redox dye. Plates were placed in an OmniLog incubator-reader at $37^{\circ} \mathrm{C}$ for 24 hours. The

141 reduction of the dye due to respiration was monitored and the results recorded as Omnilog

142 units/time.

143

144 PCR amplification and sequencing of quinolone resistance-determining region of target

145 genes

146 Primers were designed using E. aerogenes ATCC13048 (KCTC2190) (GenBank:

147 CP002824.1) as reference. For isolate 178LAV primer Ec_parE_Rev was used to amplify the

148 QRDR of parE (Table 2). Genomic DNA was extracted using a DNeasy Blood \& Tissue kit

149 (QIAGEN). PCR assays were performed as described [37]. PCR products were digested with

$1501 \mathrm{U} D p n I$ (New England Biolabs) at $37^{\circ} \mathrm{C}$ for 1 hour before being purified using the Wizard ${ }^{\circledR}$ 
151 SV Gel and PCR Clean-Up System (Promega) and sequenced (Source BioScience

152 LifeSciences).

153

\section{$154 \quad P C R$ for cat and cml genes}

155 To verify if the resistance to CHL was due to the presence of $c a t$ and/or $c m l A$ genes, a PCR 156 assay was performed [29]. The mixture was initially denaturated ( $4 \mathrm{~min}$ at $\left.94^{\circ} \mathrm{C}\right)$, followed

157 by 35 cycles of amplification ( 30 seconds of denaturation at $94^{\circ} \mathrm{C}, 30$ seconds of annealing at $15855^{\circ} \mathrm{C}$ for $\mathrm{cat}$ and $59^{\circ} \mathrm{C}$ for $\mathrm{cml}, 1$ minute of elongation at $72^{\circ} \mathrm{C}$ ) and a final elongation step (5 $159 \min$ at $72^{\circ} \mathrm{C}$ ). Amplifications were carried out on a G-Storm GS1 Thermal Cycler, UK.

160

161

Reversal of antibiotic resistance

162 Antibiotics tested were CIP and CHL, which are concerned by efflux-mediated resistance [30-

163 1]. Briefly, $100 \mu \mathrm{l}$ of MH broth was distributed into each well of the 96-wells microplate,

164 except for column 12, that contained $200 \mu$ of the highest concentration of antimicrobials.

165 Serial twofold dilutions were performed. The chemosensitisers, TZ, CPZ and NMP were

166 added at $0.5 \mathrm{x}$ MIC and PAßN at $40 \mathrm{mg} / \mathrm{L} .5 \mu \mathrm{l}$ of adjusted bacterial inoculum were inoculated

167 into the corresponding wells. Plates were incubated at $37^{\circ} \mathrm{C}$ for 18 hours. All assays were

168 conducted in triplicate in separate occasions.

169

170 Assessment of permeability/efflux activity

171 A real-time assessment of the efflux activity was conducted using a modified 96-well

172 microplate method and fluorescence readings of hoechst dye monitored with a FluoroSkan

173 Ascent Fl (Thermo Scientific, Hampshire) [32]. Efflux assays were performed by adding 20

$174 \mu 1 \mathrm{PA} \beta \mathrm{N}$ or the other chemosensitisers (at three different concentrations) to the cultures

175 monitored previously. Assays were repeated 4 times per isolate. 


\section{Modulation of the efflux dynamics - Determination of kinetic parameters}

177 To analyse the kinetics of accumulation promoted by the chemosensitisers (1/5 MIC), the rate

178 of accumulation of Hoechst dye and the time necessary to reach a plateau (designated $\mathrm{Pl}$ )

179 were determined. A regression model was applied to the accumulation of Hoechst-dye curve

180 using a logarithmic or semi-logaritmic regression (case dependent). The regression equation

181 was used to calculate the rate of fluorescence.

182

183 Statistical analysis

184 All data were analysed using Microsoft Excel and SPSS Statistics version 20.0. A dendogram 185 representing the antimicrobial susceptibility data was generated using software from Applied 186 Maths (Bionumerics, Version 7.1, Applied Math, Austin, TX).

187

188

189

190 


\section{Results and Discussion}

192 Antibiotic susceptibility testing

193 The majority of the isolates were resistant to ceftazidime and ticarcillin-clavulanic acid

194 ( $n=13)$, followed by resistance to CTX $(n=12)$ (Figure 1). Twelve isolates were also resistant

195 to azetronam. Resistance to CIP $(n=11)$ and levofloxacin $(n=10)$ was also observed.

196 Resistance to tobramycin and trimetoprim/sulfamethoxazole was recorded in 10 of the

197 isolates. Lastly, nine of the isolates were resistant to CHL. EAPOL13200 and EA131538

198 exhibited a quasi-pan resistant phenotype with susceptibility to POL.

199

200 MIC for selected chemosensitisers

201 The chemosensitisers, TZ and CPZ were the ones that showed higher inhibitory activity (MIC

$202=50$ to $>200 \mathrm{mg} / \mathrm{L}$ ) (Table 3). PABN and NMP showed lower inhibitory activity, with MIC

203 values between 250 and 2,000 $\mathrm{mg} / \mathrm{L}$ and 500 and 1,000 $\mathrm{mg} / \mathrm{L}$, respectively. The

204 chemosensitisers, OUB, VER, OME and ESO exhibited lower antibacterial activity (MIC >

$2052,000 \mathrm{mg} / \mathrm{L})$.

206

207 Assessment of respiration in the presence of chemosensitisers

208 Growth curves of all isolates were performed in the presence of varying concentrations of TZ,

209 CPZ, PAßN and NMP to ensure the chemosensitisers did not exert any effect on bacterial

210 respiration. In the presence of 200 and $400 \mathrm{mg} / \mathrm{L}$ of the chemosensitisers, there was a clear

211 impact on the respiration of E. aerogenes ATCC13048, over the course of a quasi-growth

212 curve (Figure 2). Similar effect was obtained with the other compounds. In the presence of

$213100 \mathrm{mg} / \mathrm{L} \mathrm{TZ}$ and $50 \mathrm{mg} / \mathrm{L} \mathrm{CPZ}$ there was an extension on the lag phase up to 6-7 hours

214 (Figure 2). At the highest concentrations of PAßN (400, 200 and $100 \mathrm{mg} / \mathrm{L})$, there was a

215 notable reduction in respiration, with similar effects being observed in the presence of varying 
216 concentrations of NMP (Figure 2). Importantly, concentrations of these same

217 chemosensitisers used for the reversal assays, did not exert any influence on the respiration of

218 E. aerogenes ATCC13048 (Figure 2).

219

220 In the presence of $200 \mathrm{mg} / \mathrm{L} \mathrm{TZ}$, isolates EA178LAV, EA122554, EA22SP84, and EA23214

221 exhibited a delay in the lag phase. After several hours, the respiration was re-established

222 (Figure 3). Similar results were previously reported for Salmonella, suggesting a process of 223 adaptation occurring among specific isolates [33].

224

225 A clear inhibition of bacterial respiration was obtained for the majority of the isolates at 320

226 and $160 \mathrm{mg} / \mathrm{L} \mathrm{PAßN}$ (Figure 4). $80 \mathrm{mg} / \mathrm{L}$ PAßN elicit a delay in the lag phase of EACRO,

227 EAART15737, EA178LAV, EA108418, EAPOL13200, EA23214 and EA118259 (Figure 4

228 A-E, I, J and K). After 5 hours or more of exposure to $40 \mathrm{mg} / \mathrm{L}$ PAßN a more pronounced

229 effect on the respiration of EACRO, EA108418, EA122554, EAPOL13200 and EA131538

230 (Figure 4 A, D, E, H, L) was obtained. The same was obtained with $20 \mathrm{mg} / \mathrm{L}$ PAßN for

231 isolates EA137454, EA149399, EAPOL13200 and EA131538 (Figure 4 F, G, H, L).

232 When NMP was added at the highest concentrations there was a clear inhibition on the

233 respiration of all isolates (Figure 5). At a concentration of $250 \mathrm{mg} / \mathrm{L}$ only EAART15737

234 seemed to be affected (Figure 5B). Isolates EA149399, EAPOL13200, EA23214, EA118259

235 and EA131538 showed a delay on the lag phase when incubated in the presence of $125 \mathrm{mg} / \mathrm{L}$

236 NMP (Figure 5 G, H, J, K, L).

237

238 Selection of antibiotic resistant strains and synergy testing

239 Based on the resistance profiles and considering the MIC values obtained for CIP and CHL,

240 twelve isolates were selected for further study (Table 1). Isolate EA149399 was only tested 
241 for reversal of resistance to CHL due to its susceptibility to the fluoroquinolones tested. CIP

242 and CHL were chosen to assess the possible contribution of efflux to the resistant profile of

243 the isolates [14]. All isolates (with the exception of EA149399) had MIC values of CIP

244 between 4 and $64 \mathrm{mg} / \mathrm{L}$. Similarly, for CHL values were between 128 and $>512 \mathrm{mg} / \mathrm{L}$ when

245 compared with the reference strain $(4 \mathrm{mg} / \mathrm{L})$.

246

247 Modulation of antibiotic resistance

248 The effect on resistance level to CIP and CHL was assessed in the presence of CPZ, PAßN 249 and NMP (Table 4).

250

251 In the presence of CPZ, CPZ and PAßN, isolate EAPOL13200 had an MIC for CHL of 16 $252 \mathrm{mg} / \mathrm{L}(>10 \times$ reduction in the original MIC $>512 \mathrm{mg} / \mathrm{L})$. In the presence of NMP, decrease of 253 resistance to $\mathrm{CHL}$ was also obtained with an $8 \times$ reduction in the original MIC (from $>512$ $254 \mathrm{mg} / \mathrm{L}$ to $32 \mathrm{mg} / \mathrm{L}$ ). Isolate EAART15737 showed a reversal of resistance to CHL (MIC from $255>512 \mathrm{mg} / \mathrm{L}$ to $16 \mathrm{mg} / \mathrm{L}$ ) in the presence of CPZ, PAßN and NMP.

257 The isolate EA137454 exhibited a reduction of $4 \times$ in the MIC of CIP in the presence of CPZ. 258 In this same isolate, a total reversal of resistance to CHL was observed in the presence of the 259 chemosensitisers. The MIC of CHL was strongly reduced when CPZ, PAßN and NMP were 260 present (reduction $>16 \times$ ). Since there was a total reversal of resistance to CIP and CHL in the 261 presence of CPZ, it is possible that this isolate has intrinsic mechanisms of resistance that are 262 susceptible to this compound.

264 For the remaining isolates no significant reductions in the MIC of CHL ( $\geq 512 \mathrm{mg} / \mathrm{L})$ were 265 observed (Table 4). PCR assays to detect cat [encoding a chloramphenicol acetyltransferase] 
and $c m l A$ [encoding a phenicol-specific efflux pump] were negative, failing to yield an

267 amplicon from any of the corresponding resistant isolates. Therefore, we can rule out a direct

268 contribution of both CAT and CML enzymes in this resistance. The differences in values

269 obtained for the susceptible restoration levels for EACRO, EA22SP84, EA178LAV versus

270 those obtained for EAPOL13200, EA137454 and others are likely due to other factors. The

271 divergence between the level of susceptibility restored for CHL compared to the level

272 obtained for fluoroquinolones can be associated to ability of chemosensitizers to restore

273 antibiotics susceptibility. This effect depends of the antibiotic chemical structure, their

274 respective affinity to AcrB pockets and the relative affinity of chemosensitizers for AcrB

275 pockets [34-35]. These findings highlight the differences between the isolates since not all of

276 them responded in the same way to the combinations tested suggesting difference in the

277 expression level of AcrB pump, expression of other efflux pumps, possible mutations inside

278 AcrB changing the ligand affinity, etc.

279

\section{Target gene mutations}

281 Comparative analyses were done using E. aerogenes ATCC13048 (KCTC2190) as reference.

282 Amino acid substitutions were identified in a number of target genes, associated with

283 resistance to fluoroquinolones (Table 1). Eleven isolates contained single or multiple

284 substitutions within the GyrA subunit along with either single or double substitutions in

285 GyrB. The most common amino acid substitution identified in 10 of the 11 resistant isolates,

286 was located within the gyrA-encoding subunit and was denoted as T83I (Table 1). In GyrB,

287 the most common amino acid substitution found was E466D (eight of the isolates). These

288 mutations can explain the failure of susceptibility restoration by chemosensitisers when used

289 in combination.

290 
292 All isolates were assessed for their efflux-associated MDR phenotype and ability to

293 accumulate hoechst dye (Figure 6A). In general, all isolates demonstrated the same

294 fluorescence level as the reference strain. From the four tested chemosensitisers the most

295 efficient compound promoting the accumulation of the dye was TZ, followed by NMP and

296 lastly CPZ. In the presence of glucose, there was a lower fluorescence signal detected,

297 reflecting a reduced accumulation of the dye due to the energy provided to fuel the efflux

298 machinery. Similarly, from the 3 concentrations tested for each chemosensitiser, the highest

299 (TZ $200 \mathrm{mg} / \mathrm{L} ; \mathrm{CPZ} 100 \mathrm{mg} / \mathrm{L}$ and NMP $250 \mathrm{mg} / \mathrm{L}$ ) were those that resulted in an increased

300 accumulation of the dye. This response was dose dependent. At these concentrations, addition

301 of glucose had no effect, suggesting that the efflux systems were working at saturation and

302 that respiration was impaired.

303

304 PAßN was unable to induce a significant increased accumulation of Hoechst-dye. In the

305 presence of $40 \mathrm{mg} / \mathrm{L}$ PAßN, E. aerogenes ATCC13048 and EA149399 were the only isolates

306 that showed a small rate increase in dye accumulation (Figure 6C). This observation is in line

307 with data obtained from the reversal assays wherein in the presence of PAßN, the majority of

308 the isolates did not show any increase in susceptibility to the antimicrobials tested.

309 At the highest concentration of NMP (250 mg/L) there was a noted interference/possible

310 quenching effect on the fluorescence of Hoechst-dye and this effect was more marked with

311 glucose (Figure 6B).

312

313 Modulation of the efflux dynamics - determination of kinetic parameters

$314 \mathrm{CCCP}$ is able to dissipate the proton motive force which is the energy driving force of AcrAB 315 efflux pump when the other like PAßN or NMP can block or saturate the AcrB transport for 
316 some antibiotics [36]. An effect of CCCP, PAßN and NMP on the accumulation of the dye

317 was noted with isolates EA149399, EAPOL13200, EAART15737, EA137454 and EA118259

318 (Table 5). Interestingly, the maximal restoring of CHL susceptibility was obtained with 319 PAßN-NMP on isolates EAPOL13200, EAART15737 and EA137454. This observation 320 suggests that the activity of efflux pump as transporter of CHL and dye is efficiently targeted 321 by the chemosensitisers in these isolates.

323 Regarding the mechanism and taking into account the effect of glucose, this becomes more 324 complex. Comparing the effect of PAßN alone versus $\mathrm{PA} \mathrm{N}+\mathrm{glucose}$ (or NMP alone versus $325 \mathrm{NMP}+\mathrm{glucose}$ ) with the effect of CCCP alone versus $\mathrm{CCCP}+\mathrm{glucose}$, it is clear that CCCP is 326 a real efflux energy un-coupler and its uncoupling impact can be partly restore in the presence 327 of glucose- The results obtained with PAßN, NMP and CCCP were of the same order of 328 magnitude supporting some additional effect for CPZ under these conditions. Regarding CPZ, 329 the permeabilizing effect reported for phenothiazines on biological membranes can boost the 330 observed accumulation in association with the chemical properties of Hoechst-dye [37].

331 Regarding CPZ, an effect is observed with strain EA122554. Taken together, these data 332 illustrates the impact that chemosensitisers can have on the accumulation of dyes, which can 333 be different from the effect on antibiotic activity. 


\section{Conclusion}

337 Overall, we explored the potential utility of a series of chemosensitisers in combination with

338 antimicrobials to revert antimicrobial resistance in clinical isolates of E. aerogenes. The

339 isolates studied were also characterized with target gene mutations identified. These

340 genotypes most likely underpin our inability to reverse the resistant phenotypes in the

341 presence of chemosensitisers. For some of the isolates, permeability differences were noted

342 that could contribute to their resistant profile.

343

344 Interestingly, the presence of an energy-dependent efflux of Hoescht-dye, CCCP sensitive and

345 activated by glucose, is demonstrated in strains EACRO, EA149399, EAPOL13200,

346 EAART15737, EA118259, EA137454. In strains EAPOL13200 and EA137454, PAßN can

347 impair this efflux when NMP is able to alter its activity in strains EAPOL13200 and

348 EA149399. Regarding these effects, we may hypothesize that PAßN and NMP can have same 349 activity behavior in strain EA149399 and exhibit different action in strains EAPOL13200 and

350 EA137454. These differences in the inhibitor activity may be due to different affinities of

351 Hoescht-dye, PAßN and NMP for the binding sites located inside efflux pump or by the steric 352 hindrances caused by the fixation in the AcrB pockets [38-39].

353

354 The more important point is the correlation between accumulation studies and restoration of 355 antibiotic activity. For fluoroquinolone, it is clear that the presence of target mutations in the 356 various isolates drastically impair the recovery of ciprofloxacin susceptibility whatever the 357 chemosensitizers used. About CHL, the resistance is efficiently reversed by the three 358 chemosensitizers in three strains for which an effect of CCCP on Hoescht accumulation has 359 been evidenced. This indicates that CPZ, PAßN and NMP are able to block the CHL efflux in 360 these strains and restore a partial or complete susceptible phenotype. Interestingly, no 
361 restoration of CHL susceptibility is obtained for the strain EA149399 for which an efflux

362 CCCP, PAßN and NMP sensitive is observed with Hoescht. The divergence observed

363 between the susceptibility restoration and the dye accumulation is probably due to the

364 difference of affinity and binding constant for the pump, and/or the presence of the antibiotic

365 target which initiates the bacterial death when the dye assay is only based to DNA or

366 membrane association.

367

368 This divergence dye-antibiotic must be taken into consideration when assessing the

369 contribution of efflux in clinical isolates and the evaluation of new adjuvants or

370 chemosensitizers. In this way, the use of the recent concept "structure intracellular

371 concentration activity relationship", or SICAR will be more efficient to study the correlation

372 accumulation-activity of clinically-used or new antibiotics and the potential of new

373 adjuvants/chemosensitizers [40].

374

375 Acknowledgements

376 A. Davin-Regli, J.M. Pagès, S. Fanning and M. Martins are members of the translocation 377 consortium (www.translocation.eu).

378

379 


\section{Declarations}

381 Funding: The research leading to the results discussed here was conducted as part of the

382 translocation consortium (www.translocation.eu) and has received support from the

383 Innovative Medicines joint Undertaking under Grant Agreement $n^{\circ} 115525$, resources which

384 are composed of financial contribution from the European Union's seventh framework

385 programme (FP/2007-2013) and EFPIA companies in kind contributions. This work was also

386 partly supported by grant ANR-11-BS07-019-0, Agence Nationale de la Recherche (ANR,

387 France). M.P. McCusker was partially supported by the Dawn Farm Foods Newman

388 Fellowship in Food Safety. M. Martins was partially supported by the Vétoquinol SA

389 Newman Fellowship in Food Safety.

390 Competing Interests: The authors declare that they are no conflicts of interest.

391 Ethical Approval: Not required 


\section{References}

393 [1] Wang A, Nizran P, Malone MA, Riley T. Urinary tract infections. Prim Care $394 \quad 2013 ; 40: 687-706$.

395 [2] Brooks LE, Ul-Hasan S, Chan BK, Sistrom MJ. Quantifying the Evolutionary

396 Conservation of genes encoding multidrug efflux pumps in the ESKAPE Pathogens to

397 identify antimicrobial drug targets. mSystems 2018;3(3):e00024-18.

398 [3] Davin-Regli A, Masi M, Bialek S, Nicolas-Chanoine MH, Pagès J.-M. Antimicrobial

399 resistance and drug efflux pumps in Enterobacter and Klebsiella. In: Efflux-Mediated Drug

400 Resistance in Bacteria: Mechanisms, Regulation and Clinical Implications. In Li X.-Z., Elkins

401 C. A., Zgurskaya H. I., eds. Berlin: Springer, 2016.

402 [4] Bosi C, Davin-Regli A, Bornet C, Mallea M, Pages JM, Bollet C. Most Enterobacter

403 aerogenes strains in France belong to a prevalent clone. J Clin Microbiol 1999;37:2165-9.

404 [5] Anastay M, Lagier E, Blanc V, Chardon H. Epidémiologie des bêtalactamases à spectre

405 étendu (BLSE) chez les entérobactéries dans un hôpital du sud de la France, 1999-2007.

406 Pathol Biol 2013;61:38-43.

407 [6] Vonberg RP, Wolter A, Kola A, Ziesing S, Gastmeier P. The endemic situation of 408 Enterobacter aerogenes and Enterobacter cloacae: you will only discover what you are 409 looking for. J Hosp Infect 2007;65:372-4.

410 [7] Giamarellou H. Multidrug resistance in Gram-negative bacteria that produce extended-

411 spectrum beta-lactamases (ESBLs). Clin Microbiol Infect 2005;11 Suppl 4:1-16.

412 [8] Arpin C, Dubois V, Coulange L, André C, Fischer I, Noury P, et al. Extended-spectrum

413 beta-lactamase-producing Enterobacteriaceae in community and private health care centers.

414 Antimicrob Agents Chemother 2003;47:3506-14.

415 [9] Thiolas A, Bollet C, La Scola B, Raoult D, Pagès JM. Successive emergence of

416 Enterobacter aerogenes strains resistant to imipenem and colistin in a patient. Antimicrob 
Agents Chemother 2005;49:1354-8.

418 [10] Davin-Regli A and Pagès JM. Enterobacter aerogenes and Enterobacter cloacae;

419 versatile bacterial pathogens confronting antibiotic treatment. Front Microbiol 2015;18:392.

420 [11] Malléa M, Mahamoud A, Chevalier J, Alibert-Franco S, Brouant P, Barbe J et al.

421 Alkylaminoquinolines inhibit the bacterial antibiotic efflux pump in multidrug-resistant

422 clinical isolates. Biochem J 2003;376:801-5.

423 [12] Masi M, Pagès JM, Pradel E. Production of the cryptic EefABC efflux pump in 424 Enterobacter aerogenes chloramphenicol-resistant mutants. J Antimicrob Chemother $425 \quad 2006 ; 57: 1223-6$.

426 [13] Chevalier J, Bredin J, Mahamoud A, Malléa M, Barbe J, Pages JM. Inhibitors of 427 antibiotic efflux in resistant Enterobacter aerogenes and Klebsiella pneumoniae strains. 428 Antimicrob Agents Chemother 2004;48:1043-6.

429 [14] Chevalier J, Mulfinger C, Garnotel E, Nicolas P, Davin-Régli A, Pages JM.

430 Identification and evolution of drug efflux pump in clinical Enterobacter aerogenes strains

431 isolated in 1995 and 2003; PLoS One 2008;3:e3203.

432 [15] Lavigne JP, Sotto A, Nicolas-Chanoine MH, Bouziges N, Bourg G, Davin-Regli A et al. 433 Membrane permeability, a pivotal function involved in antibiotic resistance and virulence in 434 Enterobacter aerogenes clinical isolates. Clin Microbiol Infect 2012;18:539-45.

435 [16] Doumith M, Ellington MJ, Livermore DM, Woodford N. Molecular mechanisms 436 disrupting porin expression in ertapenem-resistant Klebsiella and Enterobacter spp. clinical 437 isolates from the UK. J Antimicrob Chemother 2009;63:659-67.

438 [17] Masi M, Pagès J-M. Structure, Function and Regulation of Outer Membrane Proteins 439 Involved in Drug Transport in Enterobactericeae: the OmpF/C - TolC Case. Open Microbiol J $440 \quad 2013 ; 7: 22-33$.

441 [18] Davin-Regli A, Bolla JM, James CE, Lavigne JP, Chevalier J, Garnotel E, et al. 
442 Membrane permeability and regulation of drug 'influx and efflux' in enterobacterial

443 pathogens. Curr Drug Targets 2008;9:750-9.

444 [19] Moreillon P. Bacterial resistance to antibiotics. Schweiz Med Wochenschr

$445 \quad 1995 ; 125: 1151-61$.

446 [20] Paterson DL. Resistance in gram-negative bacteria: Enterobacteriaceae. Am J Infect

447 Control 2006;34(5 Suppl 1):S20-8.

448 [21] Kmeid JG, Youssef MM, Kanafani ZA, Kanj SS. Combination therapy for Gram-

449 negative bacteria: what is the evidence? Expert Rev Anti Infect Ther 2013;11:1355-62.

450 [22] Bohnert JA, Szymaniak-Vits M, Schuster S, Kern WV. Efflux inhibition by selective

451 serotonin reuptake inhibitors in Escherichia coli. J Antimicrob Chemother 2011;66:2057-60.

452 [23] Mahamoud A, Chevalier J, Davin-Regli A, Barbe J, Pagès J-M. Quinoline derivatives as

453 promising inhibitors of antibiotic efflux pump in multidrug resistant Enterobacter aerogenes

454 isolates. Curr Drug Targets 2006;7:843-7.

455 [24] Shiu WK, Malkinson JP, Rahman MM, Curry J, Stapleton P, Gunaratnam M, et al. A

456 new plant-derived antibacterial is an inhibitor of efflux pumps in Staphylococcus aureus. Int J

457 Antimicrob Agents 2013;42:513-8.

458 [25] Opperman TJ, Kwasny SM, Kim HS, Nguyen ST, Houseweart C, D'Souza S, et al.

459 Characterization of a novel pyranopyridine inhibitor of the AcrAB efflux pump of

460 Escherichia coli. Antimicrob Agents Chemother 2014;58:722-33.

461 [26] Societe Francaise de Microbiologie. http://www.sfm-

462 microbiologie.org/UserFiles/files/casfm/CASFMV1_0_MARS_2017.pdf

463 [27] Landman D, Georgescu C, Martin DA, Quale J. Polymyxins revisited. Clin Microbiol

$464 \quad$ Rev 2008;21:449-65.

465 [28] Lo-Ten-Foe JR, de Smet AM, Diederen BM, Kluytmans JA, van Keulen PH.

466 Comparative Evaluation of the VITEK 2, Disk Diffusion, Etest, Broth Microdilution, and 
467 Agar Dilution Susceptibility Testing Methods for Colistin in Clinical Isolates, Including 468 Heteroresistant Enterobacter cloacae and Acinetobacter baumannii Strains. Antimicrob 469 Agents Chemother 2007;51:3726-30.

470 [29] Karczmarczyk M, Abbott Y, Walsh C, Leonard N, Fanning S. Characterization of 471 multidrug-resistant Escherichia coli isolates from animals presenting at a University

472 Veterinary Hospital. Appl Environ Microbiol 2011;77:7104-12.

473 [30] Kiser TH, Obritsch MD, Jung R, MacLaren R, Fish DN. Efflux pump contribution to 474 multidrug resistance in clinical isolates of Pseudomonas aeruginosa. Pharmacotherapy $475 \quad 2010 ; 30: 632-8$.

476 [31] Fenosa A, Fusté E, Ruiz L, Veiga-Crespo P, Vinuesa T, Guallar V, et al. Role of TolC in 477 Klebsiella oxytoca resistance to antibiotics. J Antimicrob Chemother 2009;63:668-74.

478 [32] Coldham NG, Webber M, Woodward MJ, Piddock LJ. A 96-well plate fluorescence 479 assay for assessment of cellular permeability and active efflux in Salmonella enterica serovar 480 Typhimurium and Escherichia coli. J Antimicrob Chemother 2010;65:1655-63.

481 [33] Spengler G, Rodrigues L, Martins A, Martins M, McCusker M, Cerca P, et al. Genetic 482 response of Salmonella enterica serotype Enteritidis to thioridazine rendering the organism 483 resistant to the agent. Int J Antimicrob Agents 2012;39:16-21.

484 [34] Ramaswamy VK, Vargiu AV, Malloci G, Dreier J, Ruggerone P. Molecular rationale 485 behind the differential substrate specificity of bacterial RND Multi-Drug transporters. Sci Rep $486 \quad 2017 ; 7: 8075$.

487 [35] Zwama M, Yamasaki S, Nakashima R, Sakurai K, Nishino K, Yamaguchi A. Multiple 488 entry pathways within the efflux transporter AcrB contribute to multidrug recognition. Nat 489 Commun 2018;9:124.

490 [36] Nikaido H, Pagès JM. Broad-specificity efflux pumps and their role in multidrug 491 resistance of Gram-negative bacteria. FEMS Microbiol Rev 2012;36:340-63. 
492 [37] Dastidar SG, Kristiansen JE, Molnar J, Amaral L. Role of Phenothiazines and

493 Structurally Similar Compounds of Plant Origin in the Fight against Infections by Drug

494 Resistant Bacteria. Antibiotics. 2013;2:58-72.

495 [38] Vargiu AV, Nikaido H. Multidrug binding properties of the AcrB efflux pump

496 characterized by molecular dynamics simulations. Proc Natl Acad Sci U S A.

$497 \quad 2012 ; 109: 20637-42$.

498 [39] Ruggerone P, Murakami S, Pos KM, Vargiu AV. RND efflux pumps: structural

499 information translated into function and inhibition mechanisms. Curr Top Med Chem.

$500 \quad 2013 ; 13: 3079-100$.

501 [40] Masi M, Réfregiers M, Pos KM, Pagès JM. Mechanisms of envelope permeability and 502 antibiotic influx and efflux in Gram-negative bacteria. Nat Microbiol. 2017;2:17001.

503 


\section{$504 \quad$ Figure legends}

505 Figure 1: A heat-map showing the antimicrobial susceptibility profiles for E. aerogenes

506 ATCC13048 along with 19 clinical isolates (see also Table 1). The antimicrobial

507 compounds clusters are grouped according to their class.

508

509 Figure 2: Bacterial respiration trace profiles for E. aerogenes ATCC13048 alone and in 510 the presence of various concentrations of four chemosensitisers.

511 Respiration were assessed in the presence of varying concentrations of thioridazine (TZ), 512 chlorpromazine (CPZ), phenylalanine arginine $\beta$-naphthylamide (PAßN) and (D) 1-(1-

513 naphthylmethyl)-piperazine (NMP) using an Omnilog incubator-reader for 24 hours.

514

515 Figure 3: Bacterial respiration trace profiles for four clinical E. aerogenes measured in

516 the presence of various concentrations of TZ. Assays were prepared in 96-well plates in

517 media containing varying concentrations of $\mathrm{TZ}$ and also a redox dye. After innoculation with 518 the bacterial suspension, the plates were placed in the OmniLog incubator-reader at $37^{\circ} \mathrm{C}$ for

51924 hours and the reduction of the dye monitored over time. This was done for E. aerogenes 520 clinical isolates EA178LAV; EA122554; EA22SP84; EA23214 in the presence of varying 521 concentrations of TZ.

522

523 Figure 4: Bacterial respiration trace profiles for clinical $E$. aerogenes measured in the 524 presence of various concentrations of PABN. Assays were prepared in 96-well plates in 525 media containing varying concentrations of PAßN and also a redox dye. After inoculation 526 with the bacterial suspension, the plates were placed in the OmniLog incubator-reader at $37^{\circ} \mathrm{C}$ 527 for 24 hours and the reduction of the dye monitored over time. 


\section{Figure 5: Bacterial respiration trace profiles for clinical E. aerogenes measured in the}

529 presence of various concentrations of NMP. Assays were prepared in 96-well plates in

530 media containing varying concentrations of NMP and also a redox dye. After bacterial

531 inoculation, the plates were incubated in the OmniLog incubator-reader at $37^{\circ} \mathrm{C}$ for 24 hours

532 and the reduction of the dye monitored over time.

533

534 Figure 6: Accumulation of Hoechst-dye by: (A) the E. aerogenes isolates tested ; (B) E.

535 aerogenes ATCC13048 in the presence of selected chemosensitisers; (C) E. aerogenes

536 ATCC13048 and clinical isolate EA149399 in the presence of different concentrations of

537 PABN. 6A. An initial assay was conducted to evaluate the accumulation of Hoechst-dye by

538 the E. aerogenes isolates. 6B. Accumulation of Hoechst-dye by E. aerogenes ATCC13048 in

539 the presence of different concentrations of Thioridazine (TZ); Chlorpromazine (CPZ);

540 Phenylalanine arginine $\beta$-naphthylamide (PA $\beta N)$ and 1-(1-naphthylmethyl)-piperazine

541 (NMP). Cyanide-m-chlorophenylhydrazone (CCCP) was used as an internal control. Parallel

542 samples containing $0.4 \%$ glucose $(G)$ were also performed. The accumulation assay in the

543 presence of the chemosensitisers was run for 30 minutes (mins).

544

545

546

547

548

549

550 


\begin{tabular}{|c|c|c|c|c|c|c|c|c|}
\hline \multirow{2}{*}{ E. aerogenes } & \multirow{2}{*}{ Strain ID } & \multirow{2}{*}{$\begin{array}{l}\text { Biological } \\
\text { sample }\end{array}$} & \multirow{2}{*}{$\begin{array}{l}\text { Hospital of } \\
\text { origin }\end{array}$} & \multirow{2}{*}{$\begin{array}{l}\text { Year of } \\
\text { isolation }\end{array}$} & \multirow[b]{2}{*}{ gyrA } & \multicolumn{3}{|c|}{ FQ resistance genes } \\
\hline & & & & & & gyrB & parC & parE \\
\hline Reference strain & ATCC13048 & Urine & - & - & $(-)$ & $(-)$ & $(-)$ & $(-)$ \\
\hline \multirow{23}{*}{$\begin{array}{l}\text { Clinical isolates } \\
(n=19)\end{array}$} & EA23214 & Catheter & NIMES & 2005 & T83I & S464F & S80I & N477D \\
\hline & EA178LAV & Urine & MAR & 1999 & T83I & E466D & S80I & N477D \\
\hline & EA108418 & - & MAR & 2003 & T83I & E466D & S80I & N477D \\
\hline & EA137454 & - & MAR & 2003 & $\begin{array}{l}\text { A67S, } \\
\text { T83I }\end{array}$ & $\begin{array}{l}\text { L451F, } \\
\text { Q465R }\end{array}$ & S80I & N477D \\
\hline & EA122554 & - & MAR & 2003 & T83I & E466D & S80I & N477D \\
\hline & EA131538 & - & MAR & 2003 & T83I & E466D & S80I & N477D \\
\hline & EA118259 & - & MAR & 2003 & T83I & E466D & S80I & N477D \\
\hline & EABAL959 & Urine & AUB & 2002 & nd & nd & nd & nd \\
\hline & EAPOL13200 & Sputum & AUB & 2002 & T83I & E466D & S80I & N477D \\
\hline & & & & & T83Y, & & & \\
\hline & & & & & D87H, & & & \\
\hline & EAART15737 & Rectal swab & AUB & 2002 & M127L, & $\mathrm{R} 387 \mathrm{~K}$ & S80I & N477D \\
\hline & & & & & S128A, & & & \\
\hline & & & & & N200D & & & \\
\hline & EACRO & Urine & AUB & 2002 & T83I & E466D & S80I & N477D \\
\hline & EA9AB32 & Sputum & MAR & 1994 & nd & nd & nd & nd \\
\hline & EA22SP84 & Suppuration & MAR & 1995 & T83I & E466D & S80I & N477D \\
\hline & EA17AB48 & Sputum & MAR & 1995 & nd & nd & nd & nd \\
\hline & EA29AB151 & Sputum & MAR & 1995 & nd & nd & nd & nd \\
\hline & EA26UR132 & Urine & MAR & 1995 & nd & nd & nd & nd \\
\hline & EA171450 & Sputum & MAR & 2002 & nd & nd & nd & nd \\
\hline & EA132263 & Sputum & MAR & 2002 & nd & nd & nd & nd \\
\hline & EA149399 & Blood culture & MAR & 2002 & nd & nd & nd & nd \\
\hline
\end{tabular}

552 Table 1. Study collection including E. aerogenes ATCC13048 and 19 clinical isolates and

553 showing their clinical origin; geographical location, year of isolation and mutations in

554 target genes.

555 Legend. MAR - Marseille, AUB - Aubagne, NIMES - Nîmes, nd -not determined. 


\begin{tabular}{|c|c|c|c|c|c|}
\hline Target & Primer & Sequence ( 5 ' to 3 ') & $\begin{array}{c}\text { Annealing } \\
\text { temperature } \\
\left({ }^{\circ} \mathrm{C}\right)\end{array}$ & $\begin{array}{c}\text { Amplicon size } \\
\text { (bp) }\end{array}$ & Reference \\
\hline \multirow[t]{2}{*}{ gyrA } & Ea_gyrA_Fwd1 & GCTCGTATCTGGATTATGCG & 64 & 637 & This work \\
\hline & Ea_gyrA_Rev1 & CTCTTCAATACCGCGACG & & & \\
\hline \multirow[t]{2}{*}{$\operatorname{gyr} B$} & Ea_gyrB_Fwd & TACCAACAACATTCCGCAGC & 64 & 831 & This work \\
\hline & Ea_gyrB_Rev & ATGTACTGTTCCTGCTTGCC & & & \\
\hline \multirow[t]{2}{*}{$\operatorname{parC}$} & Ea_parC_Fwd & ATGCCTACCTGAACTACTCC & 64 & 701 & This work \\
\hline & Ea_parC_Rev & GTCTTCTTTCGTCCACACGG & & & \\
\hline \multirow[t]{3}{*}{ parE } & Ea_parE_Fwd & GACCGAAAGCTACGTGAACC & 64 & 875 & This work \\
\hline & Ea_parE_Rev & TCTTACGCTTCAACTGCTCC & & & \\
\hline & Ec_parE_Rev & GTTCGGATCAAGCGTGGTTT & 64 & 958 & 44 \\
\hline \multirow[t]{3}{*}{ cat } & Ec_cat_Fwd & AGT TGC TCA ATG TAC CTA TAA CC & & & \\
\hline & Ec_cat-Rev & TTG TAA TTC ATT AAG CAT TCT GCC & & & 45 \\
\hline & Ec_cml_Fwd & CCG CCA CGG TGT TGT TGT TAT C & & & \\
\hline cmlA & Ec_cml_Rev & CAC CTT GCC TGC CCA TCA TTA G & & & 46 \\
\hline
\end{tabular}

557 Table 2. Target genes, amplification primers and PCR reaction conditions used for 558 characterisation of the isolates.

559 Note: Fwd, forward; Rev, revers 


\begin{tabular}{l|c|c|c|c}
\hline \multirow{2}{*}{ E. aerogenes strains } & \multicolumn{4}{|c}{ Compounds class / MIC (mg/L) } \\
\cline { 2 - 5 } & \multicolumn{2}{|c}{ Phenothiazines } & Peptidomimetic & Piperazine \\
\cline { 2 - 5 } & TZ & CPZ & PABN & NMP \\
\hline ATCC13048 & $>200$ & 100 & 2000 & 1000 \\
\hline EACRO & 200 & 100 & 1000 & 1000 \\
\hline EA9AB32 & $>200$ & 100 & 1000 & 500 \\
\hline EA17AB48 & 200 & 100 & 1000 & 1000 \\
\hline EA29AB151 & 200 & 100 & 1000 & 1000 \\
\hline EA26UR132 & 200 & 100 & 1000 & 500 \\
\hline EA22SP84 & $>200$ & $>200$ & 1000 & 500 \\
\hline EA132263 & 200 & 100 & 500 & 500 \\
\hline EA149399 & 100 & 100 & 250 & 500 \\
\hline EA171450 & 200 & 50 & 1000 & 500 \\
\hline EAPOL13200 & 200 & 100 & 1000 & 500 \\
\hline EAART15737 & $>200$ & 100 & 1000 & 500 \\
\hline EA178 LAV & 200 & 100 & 1000 & 500 \\
\hline EABAL959 & 200 & 100 & 1000 & 500 \\
\hline EA23214 & 200 & 100 & 1000 & 500 \\
\hline EA108418 & 200 & 100 & 100 & 500 \\
\hline EA118259 & & 100 & 1000 & 500 \\
\hline EA122554 & & 100 & 1000 & 500 \\
\hline EA131538 & & & 1000 & 500 \\
\hline \multirow{2}{*yyyy}{} & & & & \\
\hline
\end{tabular}

Table 3. MIC values for selected chemosensitisers.Legend. Thioridazine (TZ);

Chlorpromazine (CPZ); 1-(1-naphthylmethyl)-piperazine (NMP); and Phe-Arg- $\beta$ naphthylamide (PAßN). Minimum Inhibitory Concentration (MIC). Based on their resistance to CIP, NAL and CHL, strains highlited in bold were selected for further synergism testing. 
Table 4: MIC values measured in the absence or in thepresence of selected chemosensitisers along with mutations in target genes.

\begin{tabular}{|c|c|c|c|c|c|c|c|c|c|c|c|c|}
\hline \multirow{2}{*}{$\begin{array}{c}\text { E. aerogenes } \\
\text { strains }\end{array}$} & \multicolumn{4}{|c|}{ CIP } & \multicolumn{4}{|c|}{ CHL } & \multicolumn{4}{|c|}{ FQ resistance genes (mutation) } \\
\hline & - & $+\mathrm{CPZ}$ & + PABN & + NMP & - & $+\mathrm{CPZ}$ & $+\mathrm{PABN}$ & + NMP & gyrA & $g y r B$ & parC & parE \\
\hline ATCC13048 & 1 & $(-)$ & $(-)$ & $(-)$ & 4 & $(-)$ & $(-)$ & $(-)$ & $(-)$ & $(-)$ & $(-)$ & $(-)$ \\
\hline EACRO & 16 & $8(2 x)$ & 16 & $8(2 x)$ & $>512$ & 512 & 512 & 512 & T83I & E466D & S80I & N477D \\
\hline EA22SP84 & 32 & 32 & $64(2 x)$ & $16(2 x)$ & $>512$ & 512 & 512 & $>512$ & T83I & E466D & S80I & N477D \\
\hline EA149399 & 1 & $(-)$ & $(-)$ & $(-)$ & 128 & 128 & 128 & 128 & NA & $\overline{N A}$ & NA & NA \\
\hline EAPOL13200 & 32 & $16(2 x)$ & 32 & $16(2 x)$ & $>512$ & $16(10 x)$ & $16(10 x)$ & $32(8 x)$ & T83I & E466D & S80I & N477D \\
\hline EAART15737 & 64 & $32(2 x)$ & $32(2 x)$ & $32(2 x)$ & $>512$ & $16(10 x)$ & $16(10 x)$ & $16(10 x)$ & $\begin{array}{c}\text { T83Y, D87H, } \\
\text { M127L, S128A, } \\
\text { N200D }\end{array}$ & R387K & S80I & N477D \\
\hline EA178LAV & 16 & $8(2 x)$ & 16 & 16 & $>512$ & 512 & 512 & 512 & T83I & E466D & S80I & N477D \\
\hline EA23214 & 32 & $16(2 x)$ & 32 & $16(2 x)$ & $>512$ & 512 & 512 & 512 & T83I & S464F & S80I & N477D \\
\hline EA108418 & 32 & $16(2 x)$ & 32 & $16(2 x)$ & $>512$ & 512 & 512 & 512 & T83I & E466D & S80I & N477D \\
\hline EA118259 & 32 & $16(2 x)$ & 32 & $16(2 x)$ & $>512$ & $256(>2 x)$ & 512 & 512 & T83I & E466D & S80I & N477D \\
\hline EA122554 & 16 & $8(2 x)$ & 16 & $8(2 x)$ & $>512$ & 512 & 512 & 512 & T83I & $\mathrm{E} 466 \mathrm{D}$ & S80I & N477D \\
\hline EA131538 & 16 & 16 & 32 & 16 & $>512$ & 512 & 512 & 512 & Т83І & E466D & S80I & N477D \\
\hline EA137454 & 4 & $1(4 x)$ & 8 & 4 & $>512$ & $1(>18 x)$ & $2(16 x)$ & $2(16 x)$ & A67S, T83I & $\begin{array}{l}\text { L451F, } \\
\text { Q465R }\end{array}$ & S80I & N477D \\
\hline
\end{tabular}

Legend. Ciprofloxacin (CIP); Chloramphenicol (CHL); (-) not determined since the isolate is susceptible to the antibiotics. NA - not applicable.

Significant differences in the MIC (considered as > 4-fold) when in the presence of the chemosensitisers are marked (bold). Isolates highlited in bold were selected for the checkerboard assay. 
Table 5. Accumulation rates measured in a Hoescht-dye uptake assay and shown as a heat-map.

\begin{tabular}{|c|c|c|c|c|c|c|c|c|}
\hline \multirow{3}{*}{$\begin{array}{c}\text { Enterobacter } \\
\text { strains }\end{array}$} & \multicolumn{8}{|c|}{ Chemosensitisers } \\
\hline & \multicolumn{2}{|c|}{ CPZ } & \multicolumn{2}{|c|}{ PABN } & \multicolumn{2}{|c|}{ NMP } & \multicolumn{2}{|c|}{ CCCP } \\
\hline & wo/Gluc & w/Gluc & wo/Gluc & w/Gluc & wo/Gluc & w/Gluc & wo/Gluc & w/Gluc \\
\hline ATCC13048 & 0.7216 & 0.4996 & 0.085 & 0.0832 & 0.1689 & 0.153 & 0.0645 & 0.1622 \\
\hline EACRO & 0.4966 & 0.378 & 0.0411 & 0.0275 & 0.0544 & 0.0204 & 0.145 & 0.0058 \\
\hline EA22SP84 & 0.5748 & 0.3964 & 0.0845 & 0.0887 & 0.1047 & 0.1042 & 0.0427 & 0.0833 \\
\hline EA149399 & 0.8903 & 0.643 & 0.0897 & 0.0207 & 0.113 & 0.0103 & 0.0969 & -0.0009 \\
\hline EAPOL13200 & 0.5009 & 0.3579 & 0.0065 & 0.0078 & 0.0844 & 0.0206 & 0.1128 & 0.0023 \\
\hline EAART15737 & 0.6169 & 0.3714 & 0.0149 & 0.0152 & -0.2135 & -0.1291 & 0.0667 & 0.0024 \\
\hline EA178LAV & 0.5871 & 0.4078 & 0.0311 & 0.0248 & 0.0561 & 0.0686 & 0.006 & 0.0719 \\
\hline EA23214 & 0.6285 & 0.2984 & 0.0093 & 0.014 & 0.018 & -0.0026 & -0.0035 & 0.0293 \\
\hline EA108418 & 0.7383 & 0.3999 & 0.0384 & 0.0606 & 0.1035 & 0.0737 & 0.0082 & 0.1094 \\
\hline EA118259 & 0.6424 & 0.3975 & 0.0804 & 0.0413 & 0.1018 & 0.169 & 0.0839 & 0.0134 \\
\hline EA122554 & 0.6755 & 0.1347 & 0.0834 & 0.0855 & 0.1274 & 0.1007 & 0.035 & 0.0832 \\
\hline EA131538 & 0.6887 & 0.4519 & 0.0326 & 0.0155 & 0.1191 & 0.0766 & 0.0072 & 0.0966 \\
\hline EA137454 & 0.7409 & 0.5719 & 0.1212 & 0.0172 & 0.1633 & 0.1546 & 0.1753 & 0.0123 \\
\hline
\end{tabular}


Legend. wo/Gluc - without glucose; w/Gluc - with glucose; Thioridazine (TZ); Chlorpromazine (CPZ); Phenylalanine arginine $\beta$-naphthylamide (PAßN); 1-(1-naphthylmethyl)-piperazine (NMP); cyanide-m-chlorophenylhydrazone (CCCP). Values are representative of the rate of accumulation of Hoescht dye in the presence of the chemosensitisers and when in the presence or absence of glucose. The grey color scheme indicates a significant effect (ratio without/with glucose >4) of energy (glucose) on the acumulation rate. 
Figure 1. A heat-map showing the antimicrobial susceptibility profiles for E. aerogenes ATCC13048 along with 19 clinical isolates (see also Table 1). The antimicrobial compounds clusters are grouped according to their class.

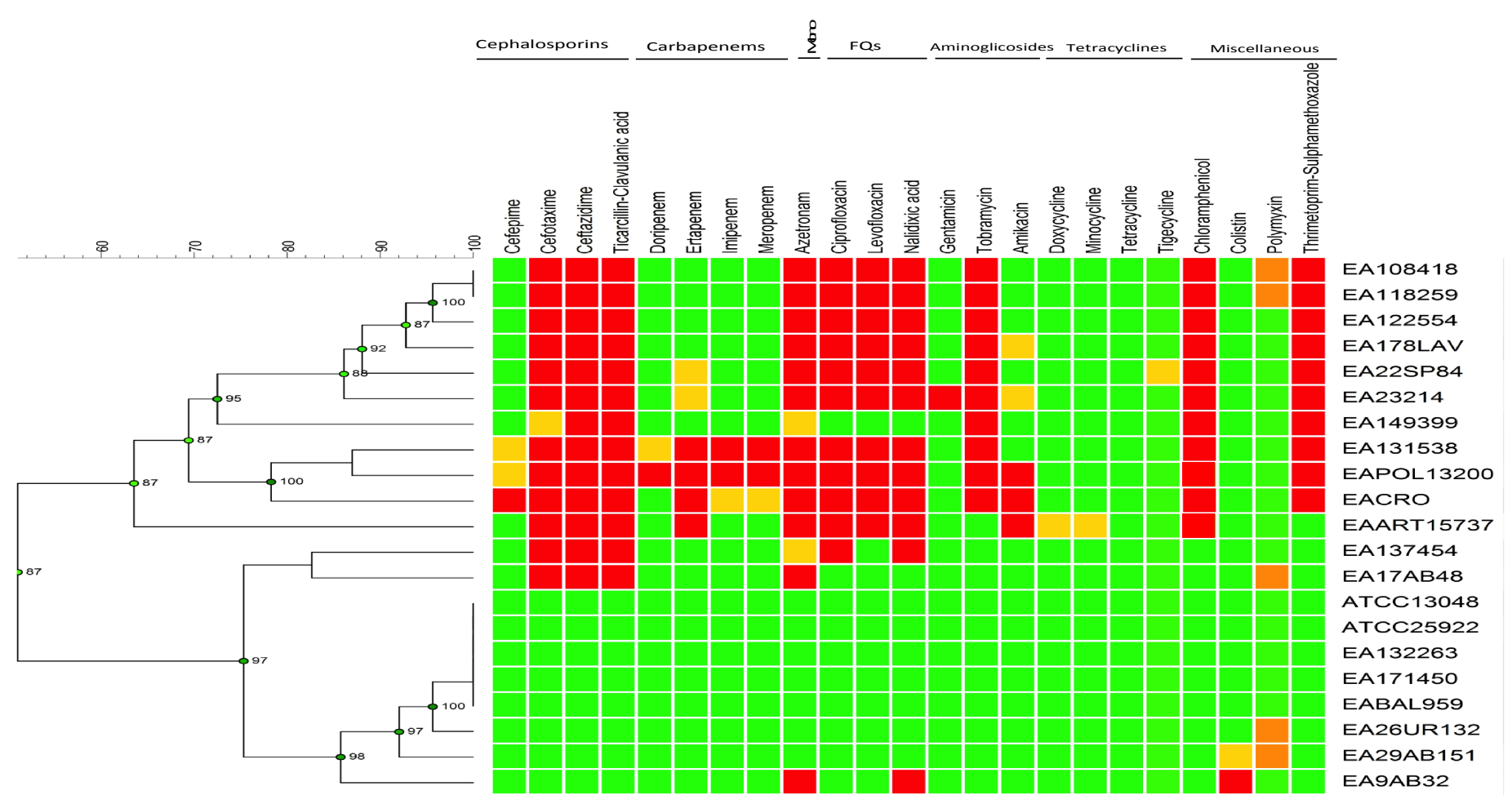


Legend of Figure 1. The dendogram was produced with software from Applied Maths (Bionumerics, Version 7.1, Applied Math, Austin, TX). Resistance profiles of the isolates are color-coded: resistant (red); intermediate (yellow/orange); susceptible (green). Penicillin G was included in the assay and analysis as an internal control (data not shown). E. coli ATCC25922 was included as quality control. 
Figure 2. Bacterial respiration trace profiles for E. aerogenes ATCC13048 alone and in the presence of various concentrations of four chemosensitisers.
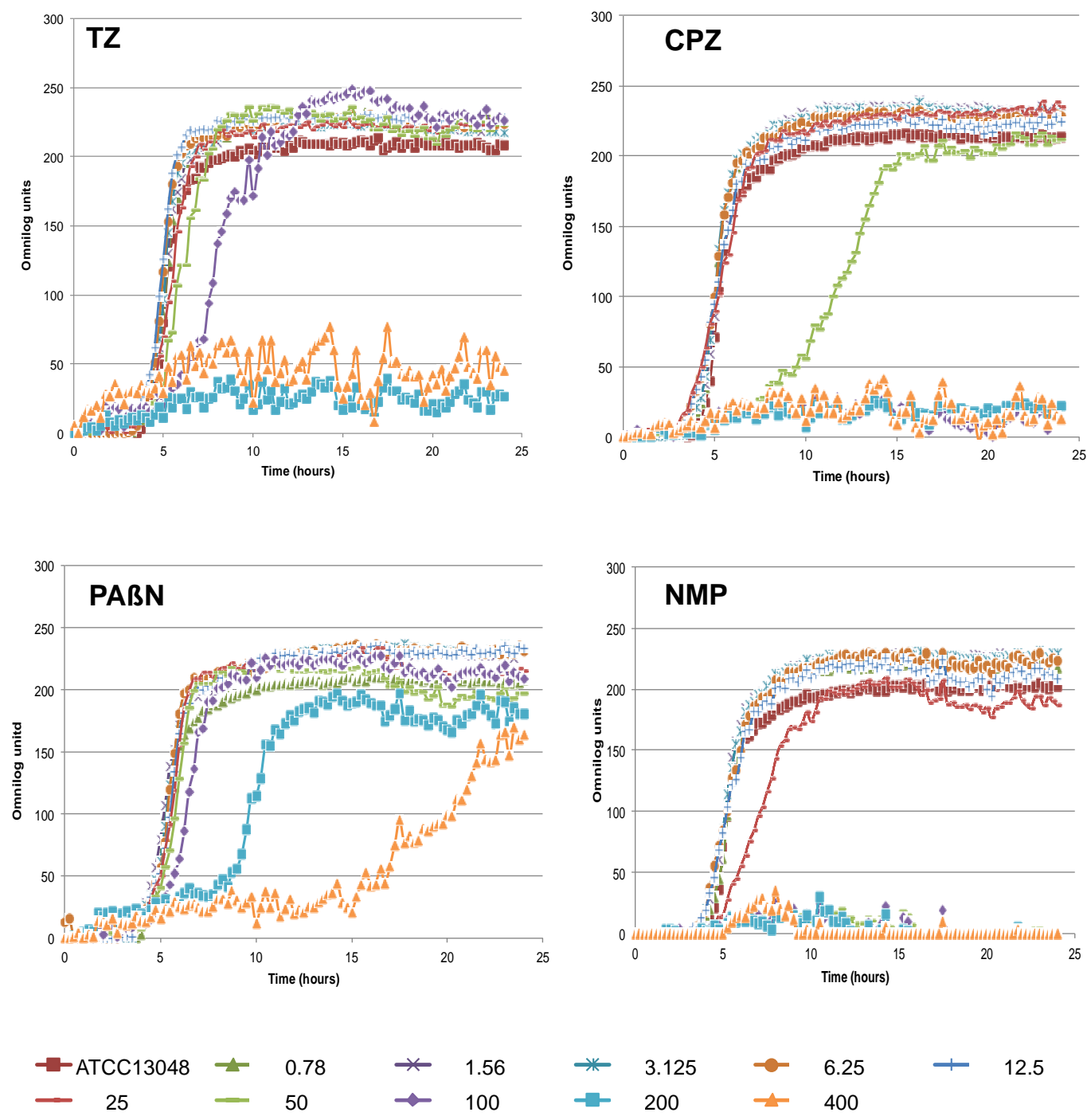

Legend of Figure 2. Respiration were assessed in the presence of varying concentrations of thioridazine (TZ), chlorpromazine (CPZ), phenylalanine arginine $\beta$-naphthylamide (PABN) and (D) 1-(1-naphthylmethyl)-piperazine (NMP) using an Omnilog incubator-reader for 24 hours. 
Figure 3. Bacterial respiration trace profiles for four clinical $E$. aerogenes measured in the presence of various concentrations of $\mathrm{TZ}$.
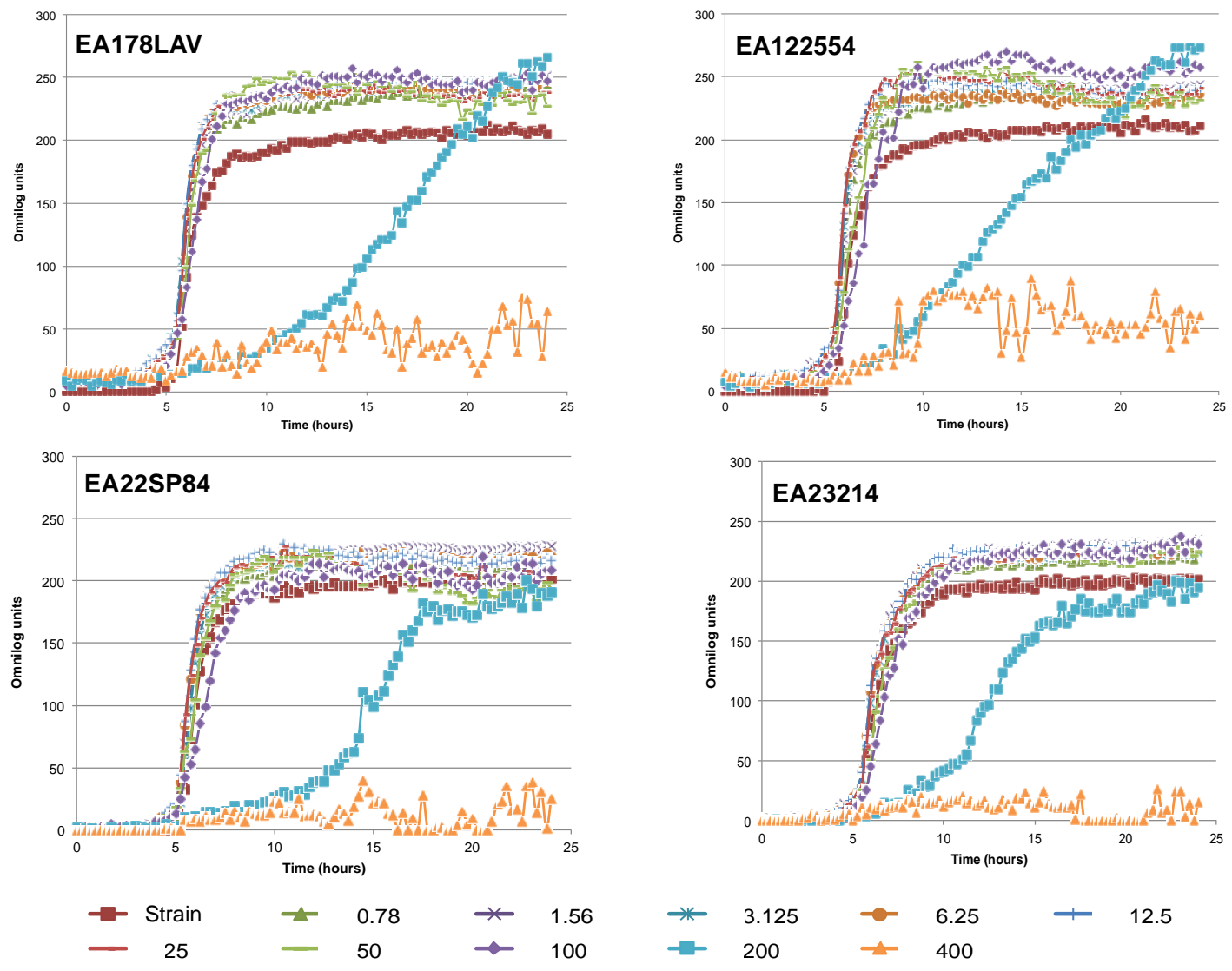

Legend of Figure 3. Assays were prepared in 96-well plates in media containing varying concentrations of $\mathrm{TZ}$ and also a redox dye. After innoculation with the bacterial suspension, the plates were placed in the OmniLog incubator-reader at $37^{\circ} \mathrm{C}$ for 24 hours and the reduction of the dye monitored over time. This was done for E. aerogenes clinical isolates EA178LAV; EA122554; EA22SP84; EA23214 in the presence of varying concentrations of TZ. 
Figure 4. Bacterial respiration trace profiles for clinical $E$. aerogenes measured in the presence of various concentrations of of PABN.
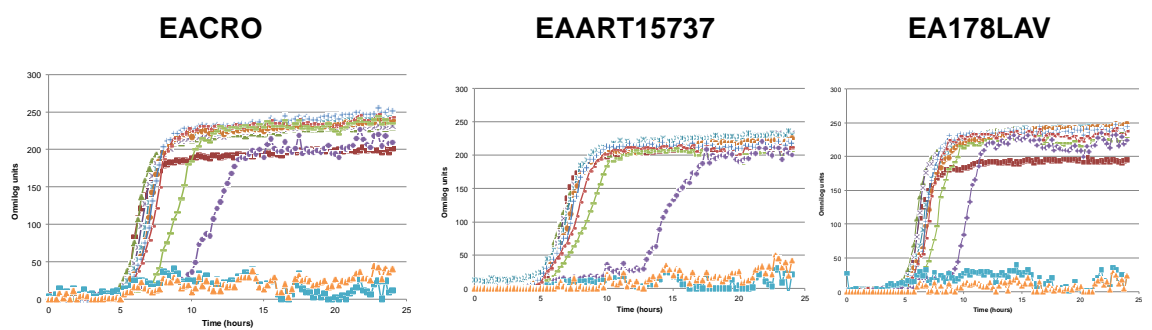

EA108418
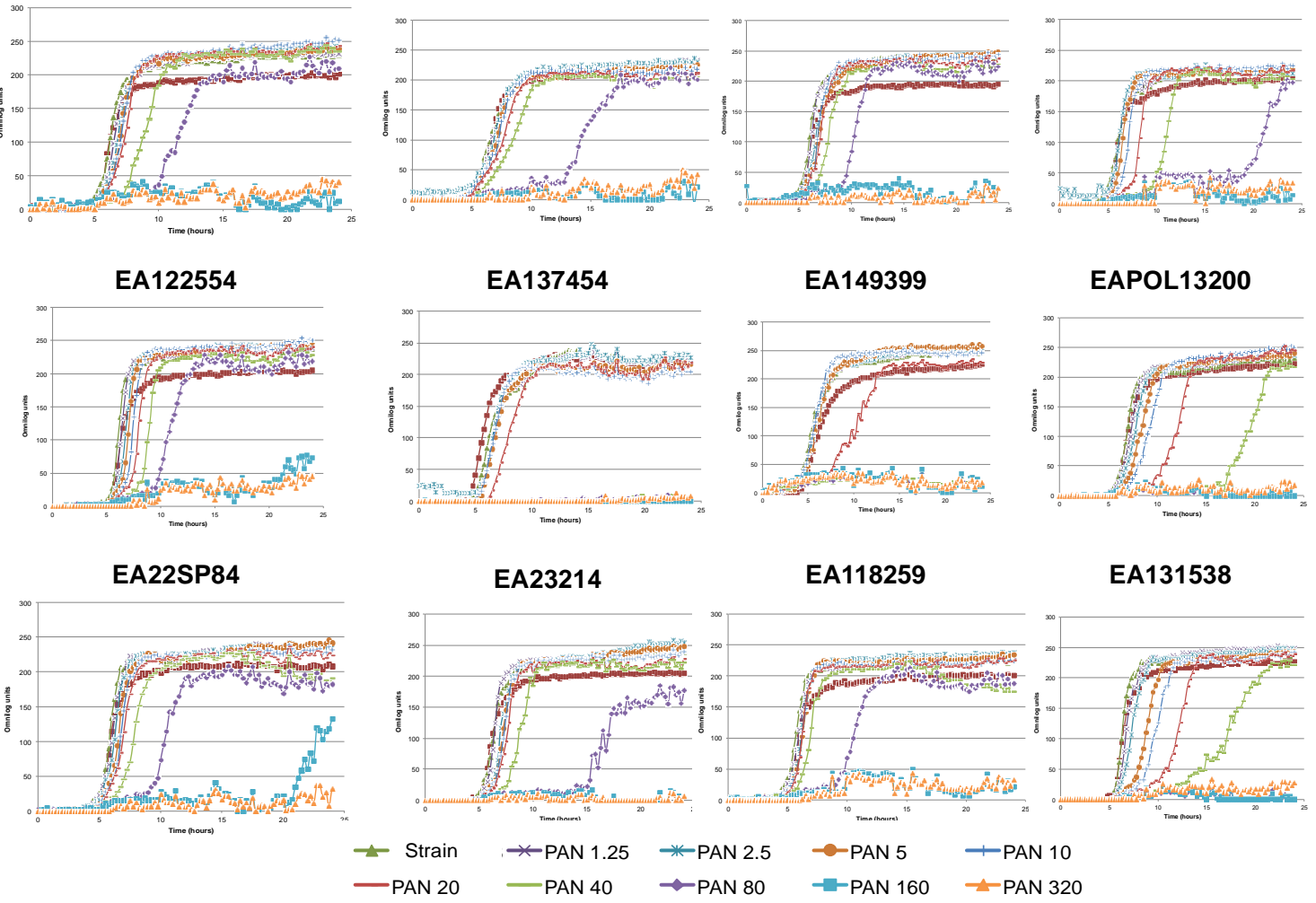

Legend of Figure 4. Assays were prepared in 96-well plates in media containing varying concentrations of PAßN and also a redox dye. After innoculation with the bacterial suspension, the plates were placed in the OmniLog incubator-reader at $37^{\circ} \mathrm{C}$ for 24 hours and the reduction of the dye monitored over time. 
Figure 5. Bacterial respiration trace profiles for clinical $E$. aerogenes measured in the presence of various concentrations of NMP.
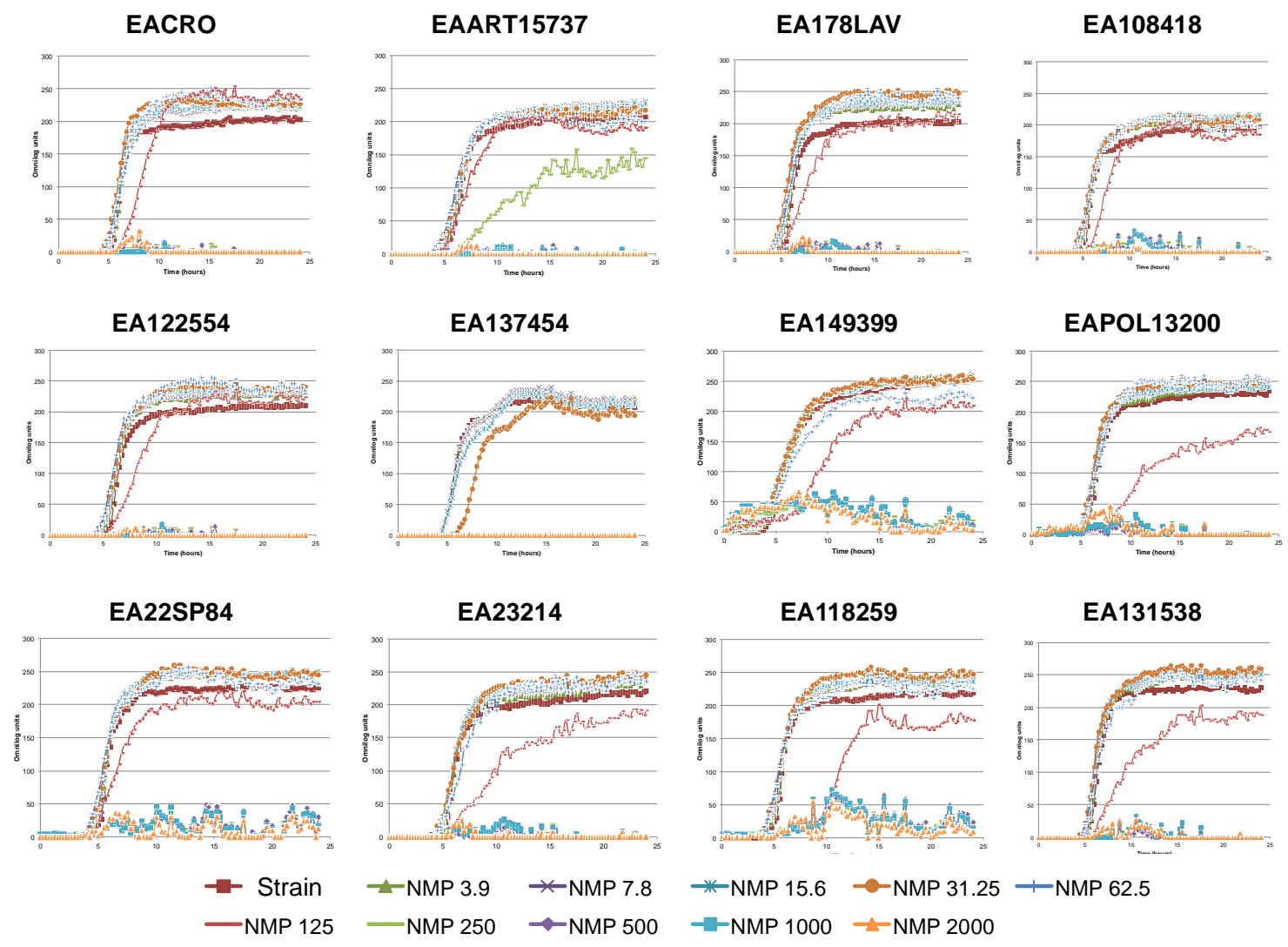

Legend of Figure 5. Assays were prepared in 96-well plates in media containing varying concentrations of NMP and also a redox dye. After bacterial innoculation, the plates were incubated in the OmniLog incubator-reader at $37^{\circ} \mathrm{C}$ for 24 hours and the reduction of the dye monitored over time. 
Figure 6 : Accumulation of Hoechst-dye by : (A) the E. aerogenes isolates tested ; (B) E. aerogenes ATCC13048 in the presence of selected chemosensitisers; (C) E. aerogenes ATCC13048 and clinical isolate EA149399 in the presence of different concentrations of PABN.

6A.

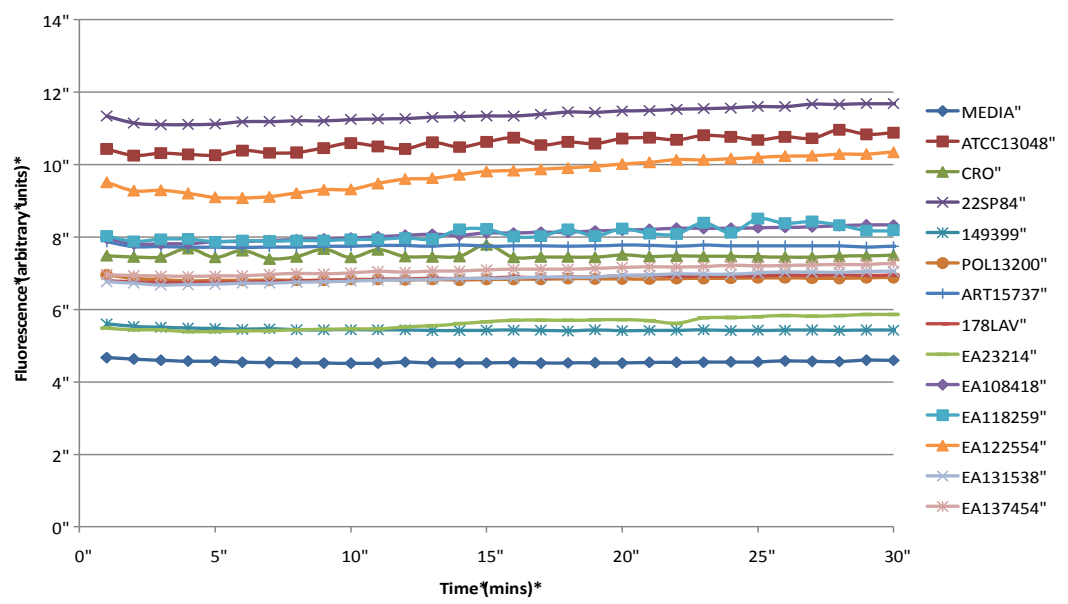

Legend of Figure 6A. An initial assay was conducted to evaluate the accumulation of Hoechst-dye by the E. aerogenes isolates. 
TZ

6B.
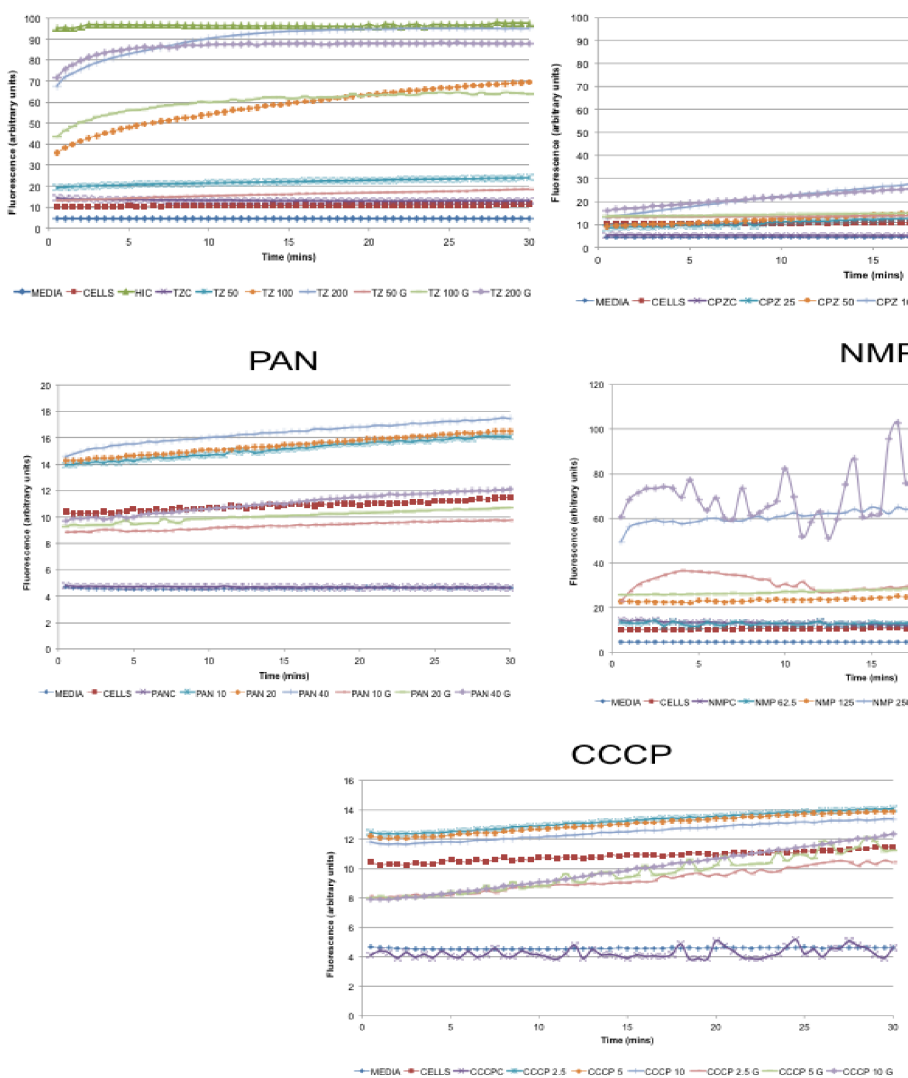

CPZ

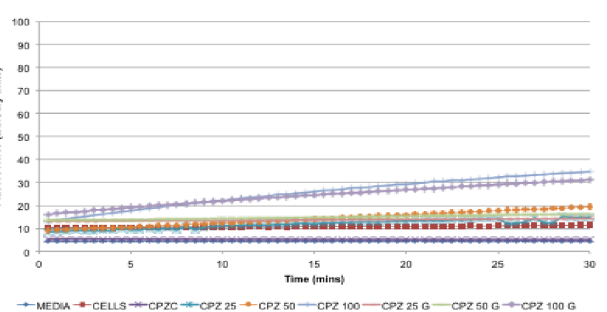

NMP

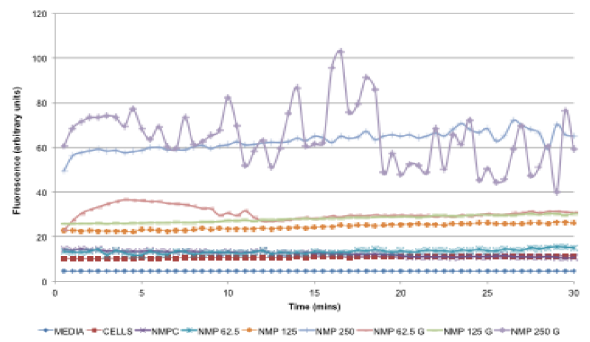

Legend of Figure 6B. Accumulation of Hoechst-dye by E. aerogenes ATCC13048 in the presence of different concentrations of Thioridazine (TZ); Chlorpromazine (CPZ); Phenylalanine arginine $\beta$-naphthylamide (PAßN) and 1-(1-naphthylmethyl)-piperazine (NMP). Cyanide-m-chlorophenylhydrazone (CCCP) was used as an internal control. Parallel samples containing $0.4 \%$ glucose $(G)$ were also performed. The accumulation assay in the presence of the chemosensitisers was run for 30 minutes (mins). 
6C.

a

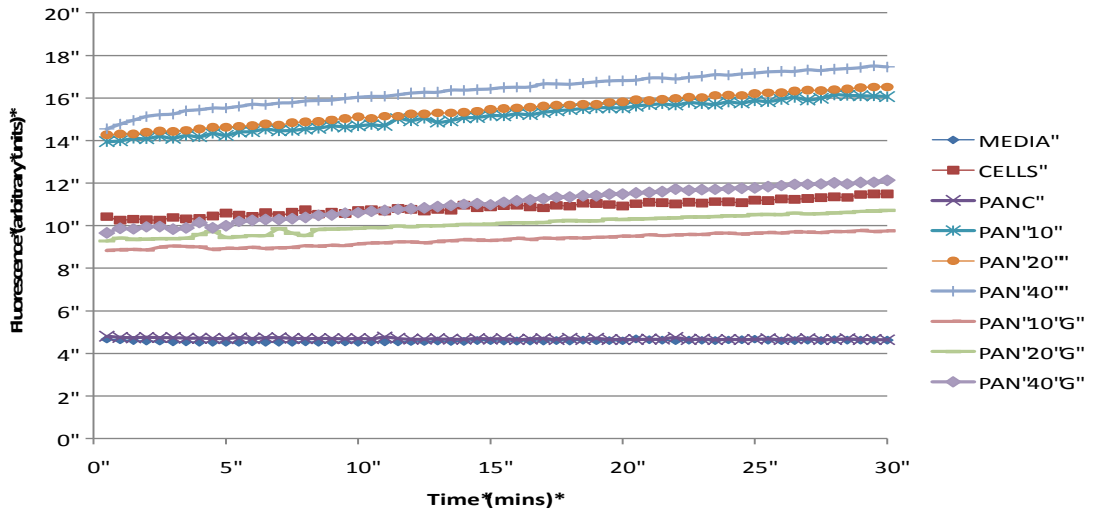

b

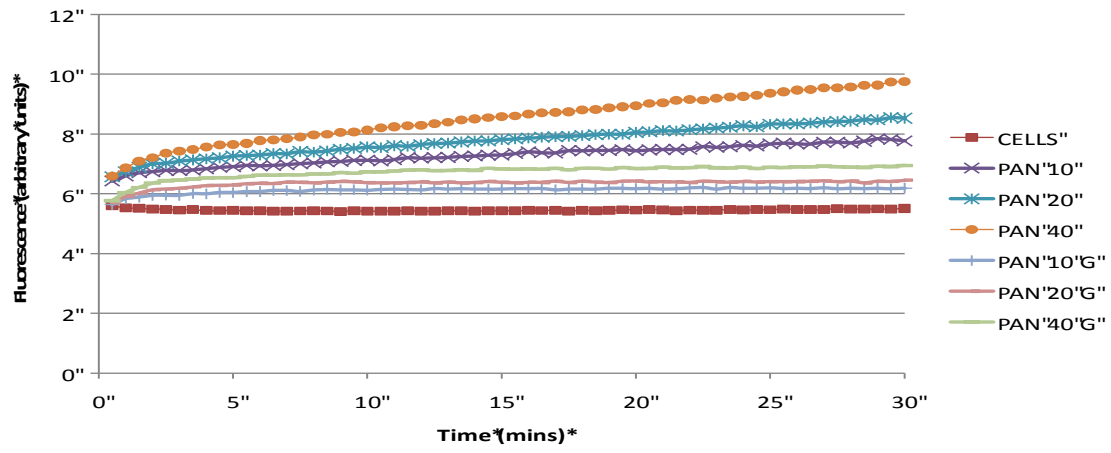

Legend of Figure 6C. Accumulation of Hoechst-dye by E. aerogenes ATCC13048 (a) and clinical isolate EA149399 (b) when in the presence of different concentrations of Phenylalanine arginine $\beta$-naphthylamide (PABN) Aliquots containing 0.4\% glucose $(G)$ were also performed in parallell. The accumulation assay in the presence of the chemosensitisers was run for 30 minutes (mins). 